\title{
Application of Distributed Parameter Model to Assessment of Glioma IDH Mutation Status by Dynamic Contrast-Enhanced Magnetic Resonance Imaging
}

\author{
Zongfang Li, ${ }^{1}$ Wei Zhao, ${ }^{1}$ Bo He, ${ }^{1}$ Tong San Koh, ${ }^{2,3}$ Yanxi Li, ${ }^{4}$ Yizhen Zeng, ${ }^{1}$ Zhuo Zhang, \\ Jingzhong Zhang, ${ }^{6,7}$ and Zujun Hou $\mathbb{1}^{6,7}$ \\ ${ }^{1}$ Department of Radiology, The First Affiliated Hospital, Kunming Medical University, Kunming 650032, China \\ ${ }^{2}$ Department of Oncologic Imaging, National Cancer Center, Singapore 169610 \\ ${ }^{3}$ Duke-NUS Graduate Medical School, Singapore 169857 \\ ${ }^{4}$ Department of Anatomical \& Cellular Pathology, The First Affiliated Hospital, Kunming Medical University, \\ Kunming 650032, China \\ ${ }^{5}$ Institute for Infocomm Research, 1 Fusionopolis Way, \#21-01 Connexis, Singapore 138632 \\ ${ }^{6}$ Suzhou Institute of Biomedical Engineering and Technology, Chinese Academy of Sciences, Suzhou 215163, China \\ ${ }^{7}$ Zhengzhou Institute of Engineering and Technology Affiliated with SIBET, Zhengzhou 450001, China
}

Correspondence should be addressed to Zujun Hou; houzj@sibet.ac.cn

Received 9 August 2020; Revised 16 October 2020; Accepted 7 November 2020; Published 23 November 2020

Academic Editor: Mattia Veronese

Copyright (C) 2020 Zongfang Li et al. This is an open access article distributed under the Creative Commons Attribution License, which permits unrestricted use, distribution, and reproduction in any medium, provided the original work is properly cited.

Previous studies using contrast-enhanced imaging for glioma isocitrate dehydrogenase (IDH) mutation assessment showed promising yet inconsistent results, and this study attempts to explore this problem by using an advanced tracer kinetic model, the distributed parameter model (DP). Fifty-five patients with glioma examined using dynamic contrast-enhanced imaging sequence at a 3.0 T scanner were retrospectively reviewed. The imaging data were processed using DP, yielding the following parameters: blood flow $F$, permeability-surface area product PS, fractional volume of interstitial space $V e$, fractional volume of intravascular space $V p$, and extraction ratio $E$. The results were compared with the Tofts model. The Wilcoxon test and boxplot were utilized for assessment of differences of model parameters between IDH-mutant and IDH-wildtype gliomas. Spearman correlation $r$ was employed to investigate the relationship between DP and Tofts parameters. Diagnostic performance was evaluated using receiver operating characteristic (ROC) curve analysis and quantified using the area under the ROC curve (AUC). Results showed that IDH-mutant gliomas were significantly lower in $F(P=0.018)$, PS $(P<0.001), V p(P<0.001), E(P<0.001)$, and $V e(P=0.002)$ than IDH-wildtype gliomas. In differentiating IDH-mutant and IDH-wildtype gliomas, $V p$ had the best performance (AUC $=0.92$ ), and the AUCs of PS and $E$ were 0.82 and 0.80 , respectively. In comparison, Tofts parameters were lower in $K^{\text {trans }}$ $(P=0.013)$ and $V e(P<0.001)$ for IDH-mutant gliomas. No significant difference was observed in Kep $(P=0.525)$. The AUCs of $K^{\text {trans }}, V e$, and $K e p$ were $0.69,0.79$, and 0.55 , respectively. Tofts-derived $V e$ showed a strong correlation with DP-derived $V e$ $(r>0.9, P<0.001) . K^{\text {trans }}$ showed a weak correlation with $F(r<0.3, P>0.16)$ and a very weak correlation with PS $(r<0.06$, $P>0.8$ ), both of which were not statistically significant. The findings by DP revealed a tissue environment with lower vascularity, lower vessel permeability, and lower blood flow in IDH-mutant than in IDH-wildtype gliomas, being hostile to cellular differentiation of oncogenic effects in IDH-mutated gliomas, which might help to explain the better outcomes in IDH-mutated glioma patients than in glioma patients of IDH-wildtype. The advantage of DP over Tofts in glioma DCE data analysis was demonstrated in terms of clearer elucidation of tissue microenvironment and better performance in IDH mutation assessment. 


\section{Introduction}

As the most common primary tumor in the brain, diffuse glioma arises from the glial cells which provide support functions to neurons and presents with high morbidity and variable outcomes [1]. The 2016 World Health Organization (WHO) classification of tumors of the central nervous system included well-established molecular signatures, such as isocitrate dehydrogenase (IDH) mutation status, expression of the transcription regulator ATRX, and 1p/19q codeletion status [2], where IDH is a small molecule protein involved in a number of cellular processes, including mitochondrial oxidative phosphorylation, glutamine metabolism, lipogenesis, glucose sensing, and regulation of cellular redox status [3-5]. IDH gene mutation testing is an important prognostic biomarker in gliomas and is relevant for glioma patient management and glioma stratification $[6,7]$. Previous studies showed that gene expression can significantly affect the disease course, and gliomas of IDHwildtype appear to rapidly acquire multiple complex genetic alterations and become glioblastomas very early in their development, and glioma patients with mutant IDH had significantly longer overall survival than patients without IDH mutation [6-10]. The prognostic importance of IDH mutation is independent of other known prognostic factors, including age, grade, and MGMT methylation status [6]. Hence, IDH mutations could serve as an ideal target of therapy, and imaging parameters are highly potential to capture the biologic complexity underlying molecular phenotypes in gliomas.

However, conventional methods for assessment of IDH mutation status are through stereotactic biopsy invasive and prone to sampling error $[11,12]$. Recently, noninvasive detection of IDH mutation status using functional imaging methods has received increasing attention [13-25]. In addition to tissue anatomic structure, functional imaging measures tissue microenvironment and provides in vivo physiologic information about brain tumors. An important functional imaging method is contrast-enhanced magnetic resonance imaging (MRI), which includes T1-weighted dynamic contrast-enhanced imaging (DCE) and T2weighted dynamic susceptibility contrast-enhanced imaging (DSC), both of which have been applied to IDH mutation assessment in gliomas [20-25].

The tissue microenvironment of frequent study includes tumor vascularity and vessel permeability. The former is modeled as cerebral blood volume (CBV) in DSC and plasma fractional volume $(V p)$ in DCE. The value of $\mathrm{CBV}$ is often normalized with respect to a reference tissue, as denoted by relative $\mathrm{CBV}$ (rCBV) or normalized $\mathrm{CBV}$ (nCBV). Among existing studies using DSC or DCE for IDH mutation status assessment, discrepancies between different studies were evident. A significantly higher rCBV in IDHwildtype compared with IDH-mutant type gliomas in all histological grades was reported in [20], whereas rCBV between IDH-wildtype and IDH-mutant gliomas did not differ significantly in histological subtypes of astrocytomas and oligodendrogliomas in [21]. Tissue vascularity was found to be significantly higher in IDH-wildtype gliomas than in IDH-mutant gliomas using DSC in [23]. However, tissue microenvironment parameters showed no correlates with glioma IDH mutation status using DCE in $[24,25]$ or DSC in [25].

The apparent conflicting results could be due to difference in imaging protocol, patient cohort, or tracer kinetic models for analyzing the acquired contrast-enhanced imaging data. Existing studies mostly utilized conventional tracer kinetic models such as the Tofts or extended Tofts model $[26,27]$, which does not differentiate the intravascular transport of tracer molecular with respect to the exchange process of tracer molecular between intravascular and interstitial spaces. There has been progress in the development of more advanced techniques in analyzing DCE data, such as the conventional compartment model (CC) [28], the adiabatic approximated tissue homogeneity model (ATH) [29], and the distributed parameter model (DP) [30]. The aforementioned two transports were separately accounted in these models, where blood flow $(\mathrm{F})$ is utilized to characterize the intravascular transport and permeability-surface area product (PS) to describe the exchange between intravascular and interstitial spaces. In comparison, these two transports are modeled using one parameter, transfer constant $\left(K^{\text {trans }}\right)$, in the Tofts or extended Tofts model. Interested readers could refer to $[31,32]$ for a review on the topic.

So far, few studies have been carried out on the investigation of these advanced tracer kinetic models in glioma molecular subtype characterization. Because advanced tracer kinetic models provide more realistic description of tracer transport in tissue microenvironment, it is expected that the derived parameters could be more interpretable with respect to tumor tissue microenvironment. This study hypothesizes that IDH mutations reduce the enzymatic activity of the encoded protein [7], leading to change in tissue microenvironment, and parameters derived using advanced tracer kinetic models would be more closely associated with glioma molecular signatures. Using DP as example, this study attempts to explore its application to glioma IDH mutation differentiation.

\section{Materials and Methods}

2.1. Subjects. This retrospective study was approved by the institutional review board. Sixty-one patients were included in this study between August 2017 and September 2019. All patients diagnosed with gliomas of grade II-IV according to the 2016 WHO guideline on brain tumor classification after craniotomy and tumor resection. Patients in the study did not have a history of previous surgery for brain tumor. Six patients were excluded due to inadequate MRI quality. A total of 55 patients (23 men, 32 women; age range, 25-72 years; mean age, $46.45 \pm 10.23$ years) were included in the study. There were 7 oligodendrogliomas (WHO grade II), 11 astrocytomas (WHO grade II), 2 anaplastic oligodendrogliomas (WHO grade III), 8 anaplastic astrocytomas (WHO grade III), and 27 glioblastomas (WHO grade IV). Molecular pathological findings of IDH were determined by Sanger sequencing for IDH hotspot mutations. There were 
24 patients with IDH mutation. A representative case is given in Figure 1.

2.2. MR Imaging Acquisition. All scans were performed using a 3.0 T MRI scanner (Discovery MR750w, GE Healthcare, Milwaukee, USA). Anatomical scans included axial T1-weighted imaging $\left(\mathrm{T}_{1} \mathrm{WI}\right), \mathrm{T} 2$-weighed imaging $\left(\mathrm{T}_{2} \mathrm{WI}\right)$, and fluid-attenuated inversion recovery (FLAIR), with parameters as follows. T1WI: repetition time (TR)/echo time (TE)/inversion time (TI) $(2182 \mathrm{~ms} / 22.7 \mathrm{~ms} / 753 \mathrm{~ms})$, field of view $(\mathrm{FOV})\left(220 \times 220 \mathrm{~mm}^{2}\right)$, matrix $(320 \times 256)$, and slice thickness (5 mm); T2WI: TR/TE (4879 ms/116 ms), FOV $\left(220 \times 220 \mathrm{~mm}^{2}\right)$, matrix $(416 \times 416)$, and slice thickness (5 mm); and FLAIR: TR/TE/TI (9000 ms/94 ms/ $2474 \mathrm{~ms})$, FOV $\left(220 \times 220 \mathrm{~mm}^{2}\right)$, matrix $(256 \times 256)$, and slice thickness $(5 \mathrm{~mm})$. DCE scan was performed using an axial fast-spoiled gradient (SPGR) echo sequence, with a precontrast and a postcontrast sequence: TR/TE $(5.318 \mathrm{~ms} /$ $1.196 \mathrm{~ms})$, FOV $\left(240 \times 240 \mathrm{~mm}^{2}\right)$, matrix $(256 \times 192)$, slice thickness $(5 \mathrm{~mm})$, flip angles of precontrast scan $\left(4^{\circ}, 8^{\circ}\right.$, and $\left.15^{\circ}\right)$, and postcontrast scan $\left(15^{\circ}\right)$. Ten dynamic precontrast scans were acquired for each flip angle, and the postcontrast sequence consisted of 180 dynamic scans, with temporal resolution 2 seconds. The contrast agent was Gadovist (Magnevist; Bayer Schering Pharma AG) at an injection rate of $3 \mathrm{~mL} / \mathrm{sec}$ (followed by a $20 \mathrm{~mL}$ normal saline flush) and a dose of $0.1 \mathrm{mmol} / \mathrm{kg}$ body weight.

2.3. Image Processing. All image processing was performed using a commercially available software (MItalytics, FITPU Healthcare, Singapore), where the method of the variable flip angle was utilized for estimating tissue contrast concentration. Voxel-wise fitting using Tofts and DP was applied to the concentration-time curve as derived from DCE data, yielding the following kinetic parameters: transfer constant $K^{\text {trans }}\left(\mathrm{min}^{-1}\right)$, fractional volume of extravascular extracellular space $V e(\mathrm{~mL} / 100 \mathrm{~mL})$, efflux rate constant Kep $\left(\mathrm{min}^{-1}\right.$, which is actually derived as the ratio between $K^{\text {trans }}$ and $V e$ ) in Tofts, and fractional volume of intravascular space $V p(\mathrm{~mL} / 100 \mathrm{~mL})$, blood flow $F(\mathrm{~mL} / \mathrm{min} / 100 \mathrm{~mL})$, permeability-surface area product PS $(\mathrm{mL} / \mathrm{min} / 100 \mathrm{~mL})$, extraction ratio of first pass $E(\%)$, and $V e$ in DP. Tumor region of interest (ROI) was manually delineated by an experienced neuroradiologist (20 years' experience) with cross-referencing anatomical imaging, while blinded to patients' pathology results. Tumor segmentation includes both the nonenhancing and the enhancing tumor core, but obvious necrosis, cystic change, hemorrhage, large vessels, and definite perilesional edema were avoided. For completeness, the Tofts and the DP models were briefly described in the following.

Tofts can be modeled [26] by

$$
C_{\text {tiss }}(t)=C_{A}(t) \otimes K^{\text {trans }} \exp \left(-\frac{K^{\text {trans }}}{V e} t\right),
$$

where $C_{\text {tiss }}(t)$ represents the tissue tracer concentrationtime curve, $C_{\mathrm{A}}(t)$ denotes the concentration of tracer in blood plasma in a feeding artery (also called the arterial input function AIF), and $\otimes$ denotes the convolution operator.

The distributed parameter (DP) model [30] accounts for concentration gradients in the vascular and interstitial compartments, with $C_{\text {tiss }}(t)$ given by

$$
\begin{aligned}
& C_{\text {tiss }}(t)=C_{A}(t) \otimes, \\
& u(t)-u\left(t-\frac{V P}{F}\right)+ \\
&\left.u\left(t-\frac{V P}{F}\right)\left\{1-\exp \left(-\frac{\mathrm{PS}}{F}\right)\left[1+\int_{0}^{t} \exp \left(-\frac{\mathrm{PS}}{V e} \tau\right) \sqrt{\frac{\mathrm{PS}}{V e} \frac{\mathrm{PS}}{F}} \frac{1}{\tau} I_{1}\left(2 \sqrt{\frac{\mathrm{PS}}{V e} \frac{\mathrm{PS}}{F}}\right) d \tau\right]\right\}\right],
\end{aligned}
$$

where $I_{1}$ is the modified Bessel function.

The most remarkable difference between two models lies in the description of two transports of tracer molecular in tissue microenvironment, namely, the intravascular transport and the exchange between the intravascular space and the interstitial space. DP separately accounts for these two processes and uses $F$ to describe the former transport and PS to characterize the latter, whereas Tofts mixes two processes and use $K^{\text {trans }}$ to model.

The precision of the model parameters under different noise conditions has been studied using the Monte Carlo simulation approach in $[30,33,34]$. The level of uncertainty (or error) associated with the estimation of the model parameter was assessed using the coefficient of variation (CV). Using Monte Carlo simulation in various noisy conditions, CVs of estimated parameters are generally small. For the tumor, the $\mathrm{CV}$ values for all parameters improve to a more acceptable level of about $13 \%$ or less for a signal-noiseratio (SNR) of 20, where SNR is taken to be the ratio of the maximum value of $C_{\text {tiss }}(t)$ (before noise was added) and the standard deviation of noise.

2.4. Statistical Analysis. Tumor voxels of each patient were pooled together, and median values of kinetic parameters were calculated. Statistical calculations were performed 




(a)

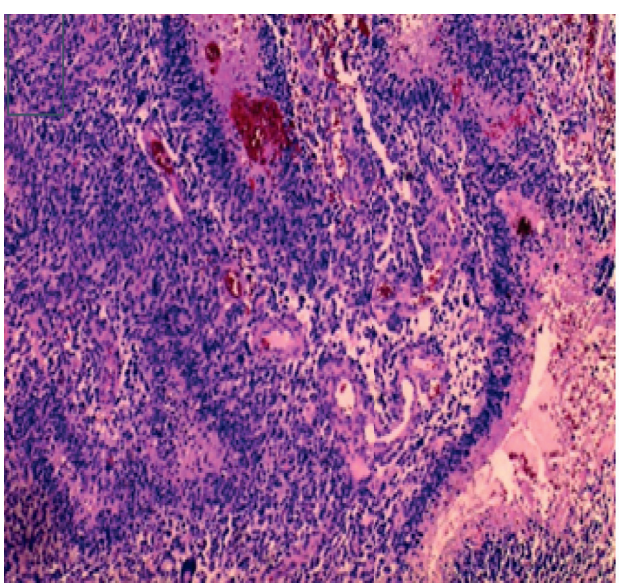

(c)

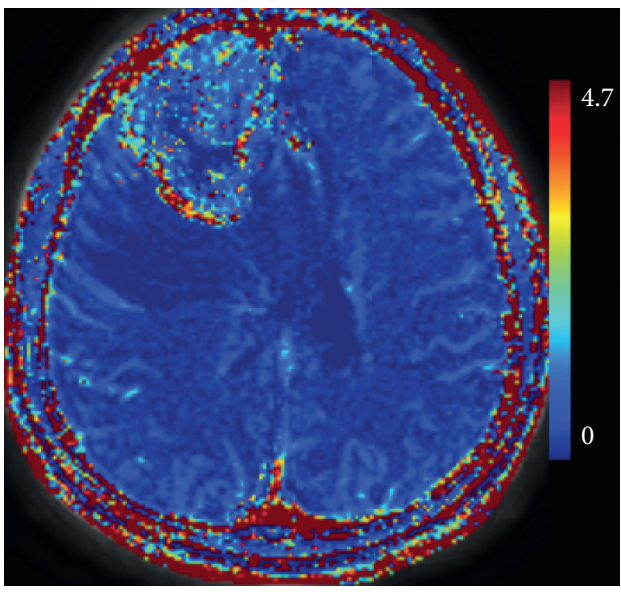

(e)

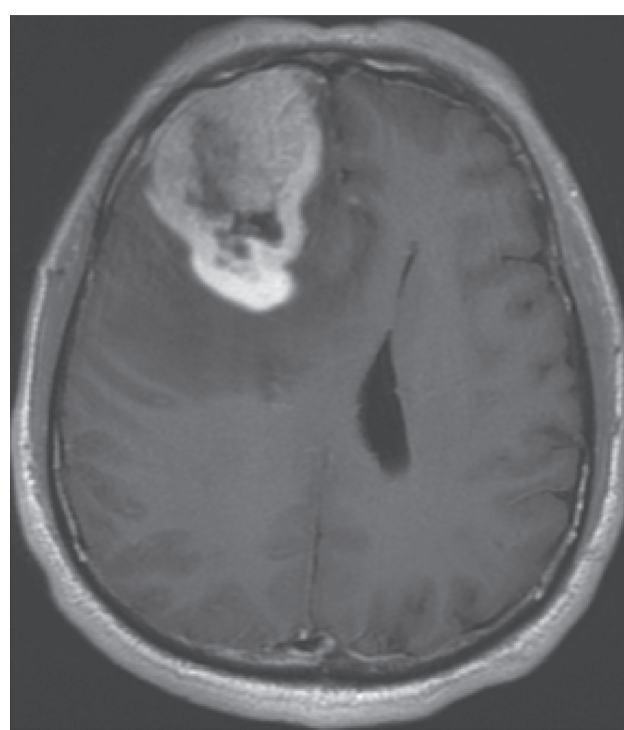

(b)

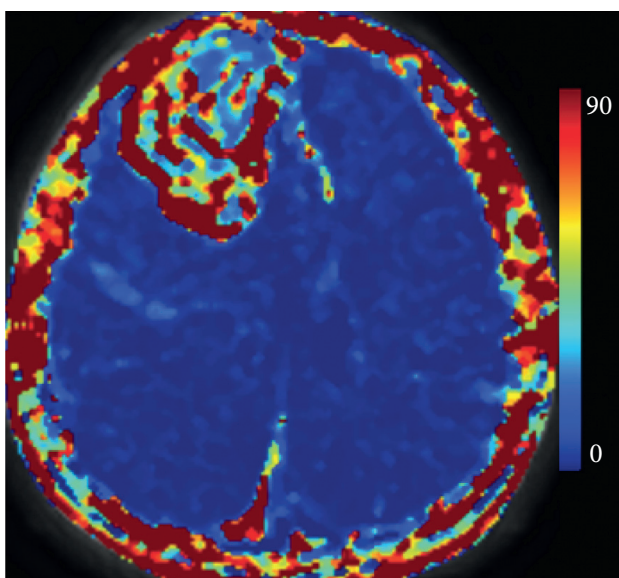

(d)

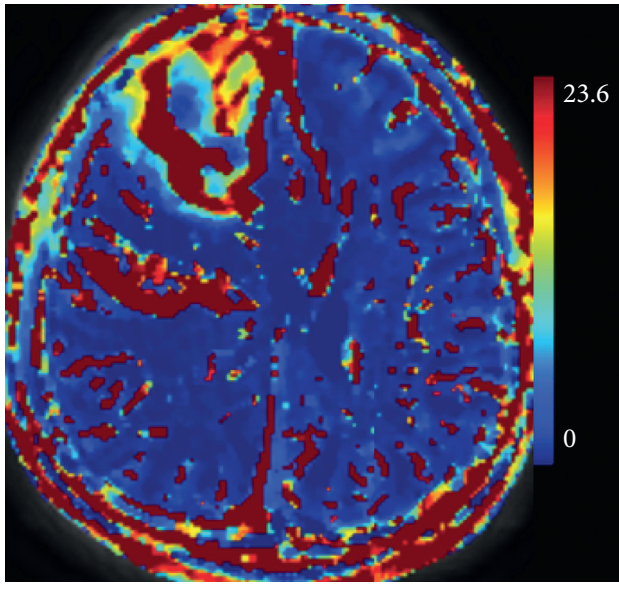

(f)

FIGURE 1: A 54-year-old male with IDH-mutant glioblastoma in the right front lobe. (a) FLAIR, showing a mass of slight hyperintensity with surrounding high signal edema in the right front lobe; (b) contrast-enhanced $\mathrm{T}_{1} \mathrm{WI}$, showing a heterogeneous contrast-enhanced mass with irregular shape; (c) histopathological image of hematoxylin-eosin stain (x10); (d) permeability-surface area product PS (mL/min/100 mL); (e) fractional volume of the intravascular space $V p(\mathrm{~mL} / 100 \mathrm{~mL})$; (f) fractional volume of the extravascular extracellular space $V e$ (mL/ $100 \mathrm{~mL}$ ) by the distributed parameter model. 
using R software (version 3.6.1). The normal distribution was tested by the Shapiro-Wilk test. A Wilcoxon test was performed to compare the medians of kinetic parameters between IDH-mutant and IDH-wildtype groups. Boxplots were employed for displaying the data dispersion and for visual comparison on the distribution of kinetic parameters in IDH-mutant and IDH-wildtype groups. Statistical significance was set at $P<0.05$. The receiver operating characteristic (ROC) curves were utilized for assessing the performance of kinetic parameters in differentiating IDHmutant from IDH-wildtype gliomas. The diagnostic performance was quantified using the area under the ROC curve (AUC). The method of Youden index was utilized for computing the optimal threshold and the corresponding sensitivity and specificity, where the optimal threshold is selected as the one to maximize the sum of sensitivity and specificity [35]. Relationship between Tofts and DP model parameters was assessed using the Spearman correlation test.

\section{Results}

3.1. Median Values of Kinetic Parameters. Table 1 summarizes the median values of kinetic parameters of two models in IDH-mutant gliomas and IDH-wildtype gliomas, the interquartile range (namely, the range between the $25^{\text {th }}$ and the $75^{\text {th }}$ percentile), as well as the results of the Wilcoxon test. The values of $K^{\text {trans }}$ and PS in IDH-mutant gliomas were smaller than in IDH-wildtype gliomas, and the difference in $K^{\text {trans }}(P=0.013)$ was less significant than in PS $(P<0.001)$. The values of $V e$ in both models in IDH-mutant gliomas are significantly smaller than in IDH-wildtype gliomas $(P<0.001$ for Tofts and 0.002 for DP). The values of $V p$ and $E$ in IDH-mutant gliomas were significantly lower than in IDH-wildtype gliomas $(P<0.001)$. Comparatively, the difference in the values of $F$ between IDH-mutant and IDHwildtype gliomas was less significant $(P=0.018)$. The difference in Tofts-derived Kep was not statistically significant $(P=0.525)$. Figure 2 shows the boxplots of DP and Tofts parameters in differentiating IDH-mutant and IDH-wildtype gliomas. The distribution of all data was not normal using the Shapiro-Wilk test at the 5\% significance level. It was of note that though a few parameters (like Tofts-derived $V e$, DP-derived PS, $V e, V p$, and $E$ ) showed a very significant difference between IDH-mutant and IDH-wildtype gliomas at the $0.1 \%$ significance level; only the distributions of DPderived $V p$ in IDH-mutant and IDH-wildtype gliomas were well separated. The large difference in data dispersion of Tofts-derived Kep between IDH-mutant and IDH-wildtype gliomas indicated low reliability of this estimated parameter.

3.2. ROC Curve Analysis. Table 2 summarizes the results of ROC curve analysis in differentiating IDH-mutant from IDH-wildtype gliomas. DP-derived $V p$ attained the best performance in discriminating IDH-mutant from IDHwildtype gliomas $(\mathrm{AUC}=0.92)$, yielding sensitivity $87.5 \%$ and specificity $80.7 \%$ with cutoff value 0.74 . Two permeability parameters PS and $E$ also showed promising discriminative power (AUC $=0.81$ and 0.80 , respectively).
Among Tofts-derived parameters, $V e$ showed the best performance in IDH mutation type differentiation with AUC 0.79. The AUCs of $K^{\text {trans }}$ and Kep were 0.69 and 0.55 , respectively. The plots of ROC curves are shown in Figure 3.

Table 3 summarizes the correlation coefficients $(r)$ between Tofts and DP model parameters in IDH-mutant and IDH-wildtype gliomas using the Spearman correlation test, where the value in bracket was the $P$ value of the test. Of first note was the not statistically significant correlation for Toftsderived $K^{\text {trans }}$ with all DP-derived parameters, and in particular, the weak correlation between $K^{\text {trans }}$ and $F(r=0.29$, $P=0.168$ in the IDH-mutant group; $r=0.24, P=0.194$ in the IDH-wildtype group) and the very weak correlation between $K^{\text {trans }}$ and PS $(r<0.01, P=0.982$ in the IDH-mutant group; $r=0.05, P=0.808$ in the IDH-wildtype group). Tofts-derived $V e$ showed a very strong correlation with DP-derived $V e$ in either IDH-mutant or IDH-wildtype gliomas $(r \geq 0.94$, $P<0.001)$, and showed a moderate to strong correlation with most other DP-derived parameters, in particular in IDH-mutant gliomas with respect to $V p$, PS, and $E(r>0.75$, $P<0.001)$.

\section{Discussion}

In this study, state-of-the-arts in DCE MRI tracer kinetic modeling were applied to the differentiation of IDH-mutant from IDH-wildtype gliomas. The former was found to be characterized with lower blood flow, lower permeability, lower $V p$, and lower Ve. The advanced tracer kinetic modeling technique was compared with the conventional Tofts model. It turned out that DP largely outperformed Tofts in IDH-mutant and IDH-wildtype differentiation.

$V p$ was found to be the most distinct feature in discriminating IDH-mutant from IDH-wildtype gliomas, with a significantly lower value in the IDH-mutant group (median, 0.21 ; interquartile range, $0.06-0.44$ ) than in the IDHwildtype group (median, 1.59; interquartile range, 0.93-2.43). Gliomas are characterized by extreme tortuosity in their capillary bed [36]. In the early stages, tumor hypoxia leads to increased expression of hypoxia-inducible factor- $1 \alpha$ (HIF-1 $\alpha$ ), which in turn mediates an increase in the vascular endothelial growth factor/vascular permeability factor (VEGF/VPF) [37, 38] that stimulates the growth of new, immature, leaky blood vessels (neovascularization). As these vessels mature, vascular intussusception and vascular cooption occurs, resulting in increased microvascular density $[38,39]$. In DCE tracer kinetic modeling, $V p$ measures the fractional volume of the intravascular space, which could be linked to the density of tissue microvasculature and is in the concept similar to CBV in the tracer kinetic modeling of DSC data. In this sense, the finding is consistent with previous studies $[23,40]$ which demonstrated that IDH-mutant tumors showed a significantly lower rCBV as compared to the wildtype counterpart. The significantly decreased $V p$ from IDH-wildtype to IDHmutant in Table 1 suggests that IDH-mutant gliomas correspond to reduced microvascular density.

In advanced tracer kinetic modeling, two types of tracer transport in tissue microenvironment are accounted for 
TABLE 1: Median value and interquartile range (i.e., range between the $25^{\text {th }}$ and the $75^{\text {th }}$ percentile, in parentheses) of kinetic parameters of two tracer kinetic models in IDH-mutant gliomas and IDH-wildtype gliomas and the results of the Wilcoxon test.

\begin{tabular}{lcccc}
\hline Model & Parameters & IDH-mutant $(n=24)$ & IDH-wildtype $(n=31)$ & $P$ value \\
\hline \multirow{3}{*}{ Tofts } & $K^{\text {trans }}\left(\mathrm{min}^{-1}\right)$ & $0.02(0.01-0.10)$ & $0.07(0.04-0.09)$ & 0.013 \\
& $V e(\mathrm{~mL} / 100 \mathrm{~mL})$ & $0.24(0.00-8.65)$ & $10.01(7.86-16.10)$ & $0.49(0.35-1.00)$ \\
& Kep $\left(\mathrm{min}^{-1}\right)$ & $0.67(0.28-70.53)$ & $12.48(10.04-14.56)$ & 0.525 \\
& $F(\mathrm{~mL} / \mathrm{min} / 100 \mathrm{~mL})$ & $9.21(4.70-12.82)$ & $3.38(2.41-5.74)$ & $<.018$ \\
DP & $P S(\mathrm{~mL} / \mathrm{min} / 100 \mathrm{~mL})$ & $0.21(0.03-1.52)$ & $23.10(16.05-36.99)$ & $<0.001$ \\
& $E(\%)$ & $1.36(0.50-10.86)$ & $9.30(5.69-15.11)$ & 0.002 \\
& $V P(\mathrm{~mL} / 100 \mathrm{~mL})$ & $0.25(0.12-9.91)$ & $1.59(0.93-2.43)$ & $<0.001$ \\
\hline
\end{tabular}

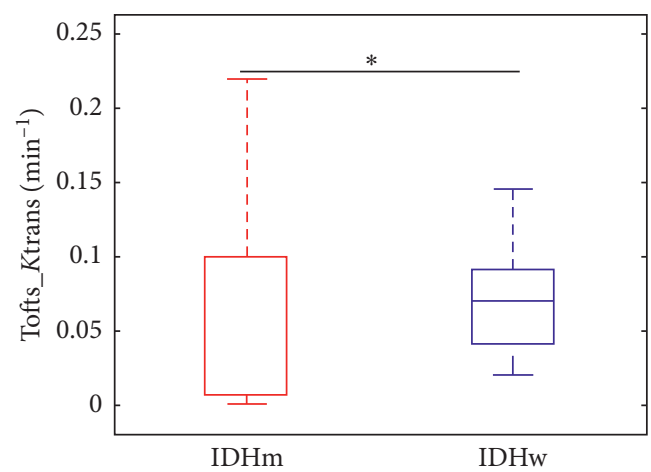

(a)

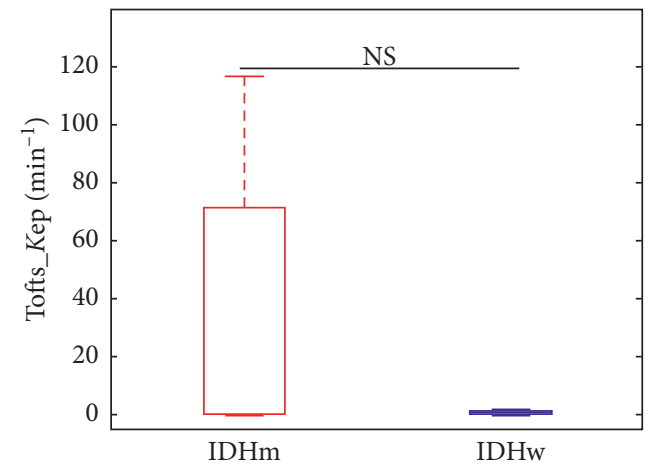

(c)



(e)

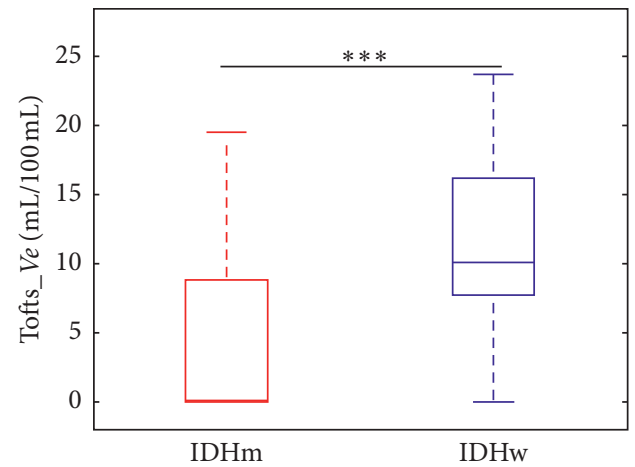

(b)

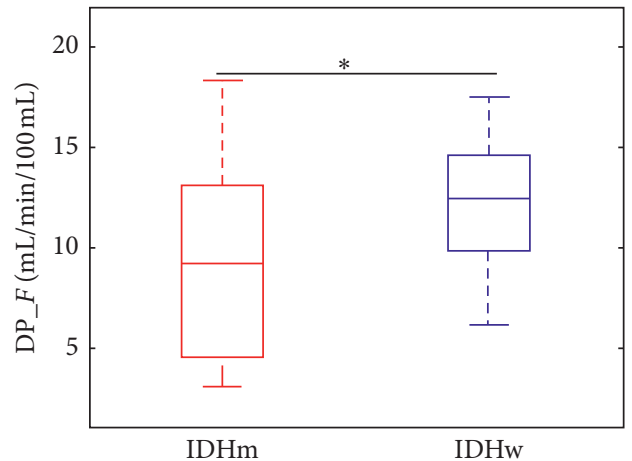

(d)

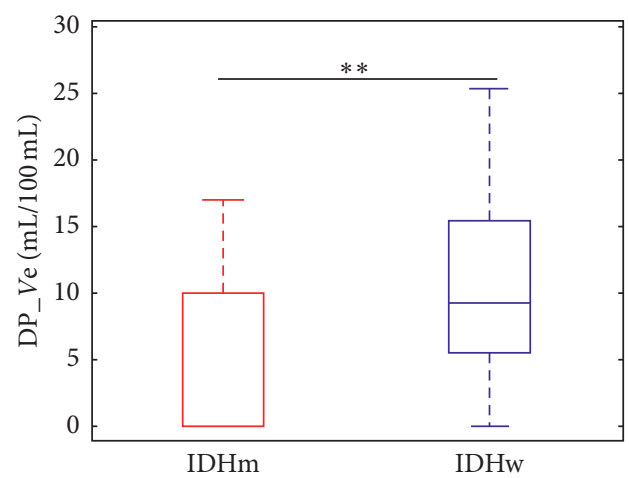

(f)

Figure 2: Continued. 


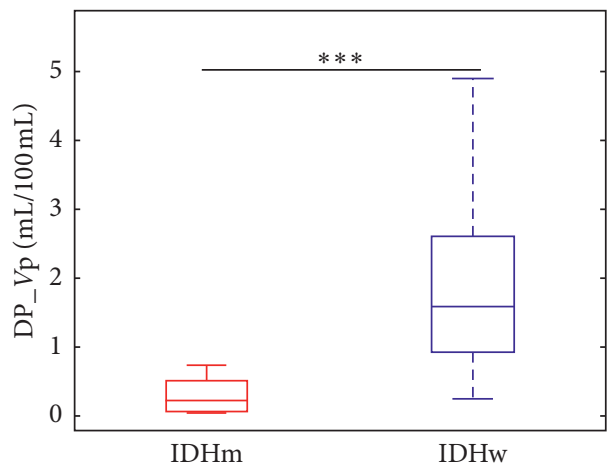

(g)

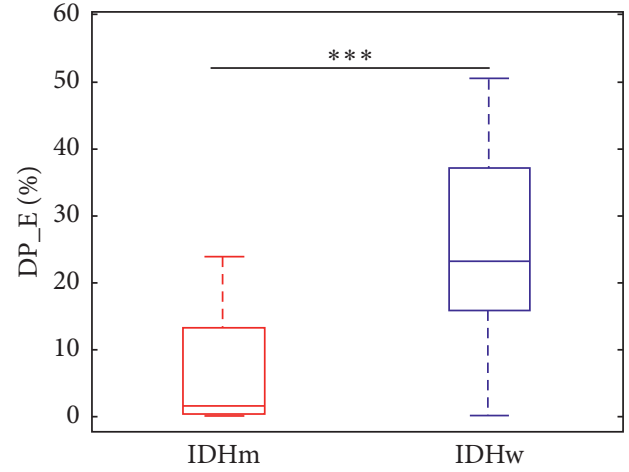

(h)

FIGURE 2: Boxplots of kinetic parameters in differentiating IDH-mutant (IDHm) with wildtype (IDHw) of gliomas, where (a)-(c) are Tofts parameters $K^{\text {trans }}, V e$, and Kep; (d)-(h) are DP parameters $F$, PS, $V e, V p$, and $E$, respectively. NS stands for not significant. ${ }^{*} p<0.05$, ${ }^{* *} p<0.01$, and ${ }^{* * *} p<0.001$.

TABle 2: Diagnostic performance of kinetic parameters of two tracer kinetic models in distinguishing IDH-mutant from IDH-wildtype gliomas.

\begin{tabular}{|c|c|c|c|c|c|c|}
\hline Model & Parameters & AUC & Cutoff value & Sensitivity (\%) & Specificity (\%) & Accuracy (\%) \\
\hline \multirow{3}{*}{ Tofts } & $K^{\text {trans }}\left(\min ^{-1}\right)$ & 0.69 & 0.02 & 50.0 & 96.8 & 72.7 \\
\hline & $V e(\mathrm{~mL} / 100 \mathrm{~mL})$ & 0.79 & 2.81 & 66.7 & 87.1 & 78.2 \\
\hline & $\operatorname{Kep}\left(\min ^{-1}\right)$ & 0.55 & 0.40 & 37.5 & 67.7 & 67.3 \\
\hline \multirow{5}{*}{ DP } & $F(\mathrm{~mL} / \mathrm{min} / 100 \mathrm{~mL})$ & 0.69 & 6.08 & 37.5 & 100.0 & 63.6 \\
\hline & $\mathrm{PS}(\mathrm{mL} / \mathrm{min} / 100 \mathrm{~mL})$ & 0.81 & 2.30 & 83.3 & 77.4 & 80.0 \\
\hline & $E(\%)$ & 0.80 & 5.98 & 70.8 & 90.3 & 81.8 \\
\hline & $V e(\mathrm{~mL} / 100 \mathrm{~mL})$ & 0.75 & 2.03 & 62.5 & 90.3 & 78.2 \\
\hline & $V p(\mathrm{~mL} / 100 \mathrm{~mL})$ & 0.92 & 0.74 & 87.5 & 80.7 & 83.6 \\
\hline
\end{tabular}

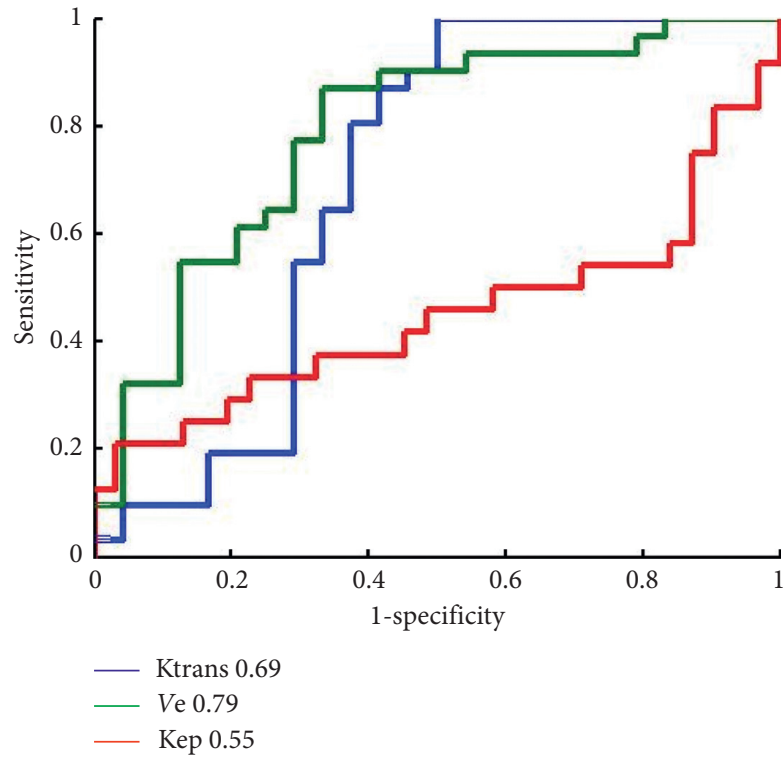

(a)

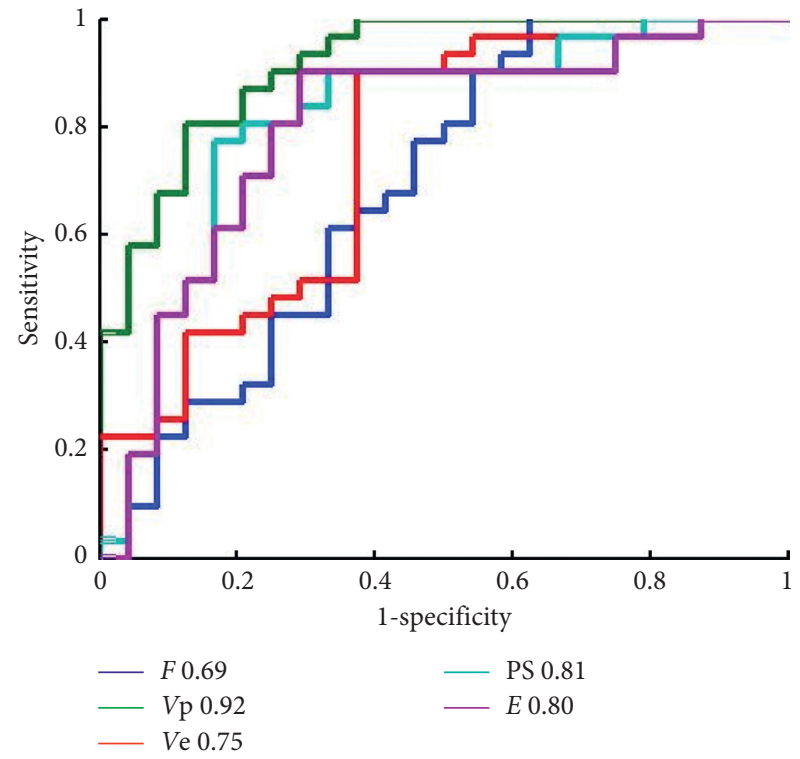

(b)

FIgURE 3: Receiver operating characteristic (ROC) plots and areas under ROC curve (AUCs) of (a) Tofts and (b) DP model parameters in differentiating IDH-mutant with IDH-wildtype of gliomas. 
TABLE 3: Results of correlation coefficient between Tofts (horizontal) and DP (vertical) model parameters in IDH-mutant and IDH-wildtype gliomas using the Spearman correlation test, where $P$ values were indicated in brackets.

\begin{tabular}{lcc}
\hline DP $\backslash$ Tofts parameters & $K^{\text {trans }}$ & $V e$ \\
\hline Mutant & & \\
$F$ & $0.29(0.168)$ & $0.34(0.105)$ \\
$V p$ & $0.06(0.790)$ & $0.78(<0.001)$ \\
$V e$ & $-0.21(0.333)$ & $0.94(<0.001)$ \\
PS & $0.00(0.982)$ & $0.83(<0.001)$ \\
$E$ & $-0.03(0.906)$ & $0.90(<0.001)$ \\
\hline Wildtype & & \\
$F$ & $0.24(0.194)$ & $0.09(0.615)$ \\
$V p$ & $0.07(0.713)$ & $0.44(0.013)$ \\
$V e$ & $-0.23(0.209)$ & $0.95(<0.001)$ \\
PS & $0.05(0.808)$ & $0.44(0.014)$ \\
$E$ & $-0.20(0.288)$ & $0.66(<0.001)$ \\
\hline
\end{tabular}

intravascular perfusion and exchange between intravascular space and extravascular space, which are explicitly described and separately characterized by the blood flow $(F)$ and permeability-surface area product (PS). E estimates the ratio of tracer leakage during the first pass and represents another permeability parameter. As shown in Table 1, the difference in $F$ between IDH-mutant and IDH-wildtype gliomas is evidently less significant than the difference in PS and $E$ $(P=0.018$ vs $P<0.001)$. As pointed out in $[39,41]$, blood flow can be extremely variable and heterogeneous in any given region of a tumor. The diagnostic power of $F$ is inferior to that of PS and $E$ in differentiating IDH-mutant from IDHwildtype gliomas (AUC $=0.69$ vs $0.81,0.80$ ).

The increased PS and $E$ from IDH-mutant to IDHwildtype indicate the increased leakiness of vessels in IDHwildtype gliomas. The leakiness of blood vessel could be affected by multiple factors, such as the luminal surface area, permeability of the vessel wall, blood flow, and hydrostatic, interstitial, and osmotic gradients across the endothelium [41-43]. To ensure the delayed permeability is measured, the postcontrast scan in this study captures not only the first pass of the contrast agent but also the bidirectional exchange between the intravascular space and interstitial space, which lasts about 6 mins in total. Interested readers can refer to $[44,45]$ for analysis of tracer transport and imaging protocol design. The good performance of PS in this study illustrates the prominent feature of leaky vessels in gliomas and justifies the separate modeling of intravascular transport and bidirectional exchange between intravascular space and extravascular space in advanced tracer kinetic modeling techniques.

Parameter $V e$ can be interpreted as the fractional volume of the extravascular extracellular space, which may reflect mitotic activity of cells. Tumor tissue is typically characterized by overgrowth of tumor cells, leading to the decreased interstitial space. In general, the higher the cells' mitotic activity, the smaller the interstitial space. Nevertheless, care should be taken in the interrogation of $\mathrm{Ve}$ in brain tumor. In our study, evident increase in $V e$ was observed in IDH-wildtype gliomas $(P=0.002$ for $\mathrm{DP})$, which is inconsistent with previous studies $[24,25]$, where no significant difference was observed on $V e$ values between IDH-mutant and IDH-wildtype gliomas. This might be due to the differences in imaging protocols and patient demography. The temporal resolution is $4 \sim 5$ seconds in [25], and the study by [24] focused on high-grade glioma with postcontrast scan time about 2 mins, which primarily captures the first pass of contrast transport. This study was performed on both low-grade and high-grade gliomas.

Nevertheless, the finding of higher $V e$ did not mean that IDH-wildtype gliomas had lower mitotic activity of cells. The apparent controversy could be explained as follows. Compared with other tumors (like liver tumor), vessel permeability is much smaller in brain tumor due to the presence of the blood-brain barrier (BBB). The exchange between intravascular space and interstitial space is a very slow process in brain tumor due to the presence of BBB. In particular, to measure the compromise of $\mathrm{BBB}$ using DCE imaging, scanning time is usually much longer than in tumor imaging, typically 20 30 minutes [46]. As such, the insufficient scan time only allows to observe the exchange process during this period and to measure the actual volume in the interstitial space as manifested by the contrast agent during this period. This "measured" interstitial space closely reflected the vessel permeability. Studies using DWI found that IDH-wildtype gliomas had lower apparent diffusion coefficient than IDH-mutant gliomas, indicating more restricted diffusion space of water molecular in IDH-wildtype gliomas [21]. Thus, the "actual" extravascular extracellular space in IDH-wildtype gliomas would be smaller than that in IDHmutant gliomas. Therefore, different from applications to most other types of solid tumor, the "measured" $V e$ in glioma DCE did not tell the mitotic activity of tumor cells, and the increased $V e$ in IDH-wildtype gliomas might indicate elevated vessel permeability in these gliomas.

This seems to be in contrast with the recent finding by Mills and colleagues [47] on glioblastoma, which shows that high values of $V e$ are significantly associated with increased mitotic activity. Nevertheless, Mills and colleagues [47] also stated that the positive correlation between $V e$ and mitotic activity was "unexpected." Furthermore, it is pointed out in [47] that there are potential modeling problems associated with the calculation of $V e$, and clearly, it can be estimated only in perfused tissue where there is significant leakage of the contrast agent. This problem means that summary statistics presented in [47] and other studies reflect only perfused tissue with contrast agent leakage. In addition, the authors [47] suggest that the relatively low dynamic sampling duration (6 minutes) will affect $V e$ estimates to some degree, which corroborates our analysis. The connection of estimated $\mathrm{Ve}$ in gliomas with vessel permeability also helps to explain the correlation between Tofts-derived Ve and DP-derived PS in Table 3, which shows a strong statistically significant correlation $(0.83, P<0.001)$ in the mutated IDH group and only a moderate statistically significant correlation $(0.44$, $P<0.001)$ in the wildtype IDH group. This is because vessels in IDH-wildtype gliomas are leakier than in IDHmutant gliomas, and the $V e$ estimates in more perfused IDH-wildtype gliomas would be more of balance between 
vessel leakage and the actual extravascular extracellular space than in IDH-mutant gliomas.

As for the Tofts model, the values of $K^{\text {trans }}$ and $V e$ in the IDH-mutant group were lower than in the IDH-wildtype group $(P=0.013,<0.001)$, and no significant difference was observed for Kep $(P=0.525)$, where $V e$ attained the best diagnostic performance with AUC 0.79. Tofts-derived $V e$ was strongly correlated with DP-derived $V e$. Though $K^{\text {trans }}$ is frequently understood as vessel permeability, the correlation with respect to DP-derived PS was very weak. The moderate performance of $K^{\text {trans }}(\mathrm{AUC}=0.69)$ could be due to the uncertainty on the physiological interpretation of $K^{\text {trans }}$, which is assumed as the mixture of the transport of the contrast agent within the vascular space and the transport of the contrast agent exchange between the intravascular space and interstitial space [48]. $K^{\text {trans }}$ describes only PS when the transport of tracer across the vessel wall is limited by permeability. When blood flow is slow, $K^{\text {trans }}$ becomes flow dependent. As a result, the measured $K^{\text {trans }}$ could be confounded by the aforementioned but unknown combination of the implicit processes in tissue microenvironment. Overall, the performance of Tofts is evidently inferior to that of DP.

In our study, a significant difference between IDHmutant and IDH-wildtype gliomas was found in perfusion parameters. Gliomas with IDH mutations manifested with reduced tissue vascularity, blood flow, and vessel permeability, indicating a hypoxic microenvironment in these tumors, which might restrict the cellular differentiation of oncogenic effects. Mutations in the IDH genes result in disruption of the enzyme's normal catalytic activity and production of 2-hydroxyglutarate (2-HG), an oncometabolite, which leads to genetic and epigenetic dysregulation and subsequent tumorigenesis [49].

A main limitation of this study is that only sample statistics of tumor ROI are taken into account in representing the corresponding patient's disease status, though the calculation of kinetic parameters is carried out over each voxel. As tumor is characterized by heterogeneity, particularly, in apparently low-grade gliomas, approximately $80 \%$ of those may contain areas of higher-grade anaplasia [50]; thus, analysis with account of parameter distribution or intervoxel information could be helpful in better understanding the underlying tumor tissue microenvironment. Another limitation is the relatively small sample size, which would influence the confidence interval of the estimated cutoff, sensitivity, and specificity. As IDH mutations were present in $80-90 \%$ of grade II and III gliomas [11, 51], it would be interesting to investigate the relationship between histologic subtypes and IDH mutation status and track the tumor evolution, which will be explored in a larger dataset in future. The required sample size in a diagnostic accuracy study has been formulated in [52] as a function of the measure of diagnostic accuracy, conjectured level of accuracy, suspected difference in accuracy between the two imaging techniques, observer variability, and ratio of patients without to patients with the condition, where a table was provided to serve as a guidance on sample size estimation in the design of the diagnostic accuracy study.
Alternatively, one might use bootstrapping by repeatedly resampling with replacement from observed data to obtain a sequence of estimates on the diagnostic accuracy and take average as the final estimate [53].

\section{Conclusions}

The distributed parameter model in dynamic contrast-enhanced imaging has been applied to glioma IDH mutation differentiation and compared with the conventional Tofts model. The results turned out that IDH-mutant gliomas were featured by low vascularity $(V p)$, low blood flow, and low vascular permeability, suggesting a tissue microenvironment in IDH-mutant gliomas hostile to the cellular differentiation of oncogenic effects. Consistent with previous DSC studies, tissue vascularity was the most prominent for characterizing glioma IDH mutation, with good potential as imaging surrogate for molecular features of gliomas. Besides that, DCE provided additional information on vessel permeability, which also presents a good diagnostic performance in differentiating IDH-mutant from IDH-wildtype gliomas. On top of that, glioma DCE differed from DCE studies of most other types of solid tumor in the lack of clues on cellular mitotic activity due to the presence of the bloodbrain barrier.

\section{Data Availability}

The availability of the medical data used in the study is subject to legal and ethical policy on patients' privacy in the People's Republic of China.

\section{Conflicts of Interest}

The authors declare that there are no conflicts of interest regarding the publication of this paper.

\section{Acknowledgments}

This work was supported by Yunnan Provincial Science and Technology Department, Kunming Medical University applied basic research (2019FE001(-052)), and Yunnan Provincial Health Science and Technology Program (2018NS0120). The authors appreciate the assistance of Mr. Liuyang Chen and Mrs. Zibo Chen (Fisca Healthcare Ltd, Kunming, China) in software customization for specific applications in this study.

\section{References}

[1] A. Omuro and L. M. DeAngelis, "Glioblastoma and other malignant gliomas," JAMA, vol. 310, no. 17, pp. 1842-1850, 2013.

[2] D. N. Louis, A. Perry, G. Reifenberger et al., "The 2016 World Health Organization classification of tumors of the central nervous system: a summary," Acta Neuropathologica, vol. 131, no. 6, pp. 803-820, 2016.

[3] D. C. LaPorte and D. E. Koshland, "Phosphorylation of isocitrate dehydrogenase as a demonstration of enhanced sensitivity in covalent regulation," Nature, vol. 305, no. 5932, pp. 286-290, 1983. 
[4] Z. J. Reitman and H. Yan, "Isocitrate dehydrogenase 1 and 2 mutations in cancer: alterations at a crossroads of cellular metabolism," JNCI Journal of the National Cancer Institute, vol. 102, no. 13, pp. 932-941, 2010.

[5] M. Ständer, A. Peraud, B. Leroch, and F. W. Kreth, "Prognostic impact ofTP53 mutation status for adult patients with supratentorial World Health Organization Grade II astrocytoma or oligoastrocytoma," Cancer, vol. 101, no. 5, pp. 1028-1035, 2004.

[6] M. Sanson, Y. Marie, S. Paris et al., "Isocitrate dehydrogenase 1 codon 132 mutation is an important prognostic biomarker in gliomas," Journal of Clinical Oncology, vol. 27, no. 25, pp. 4150-4154, 2009.

[7] H. Yan, D. W. Parsons, G. Jin et al., "IDH1 and IDH2 mutations in gliomas," The New England Journal of Medicine, vol. 360, no. 8, pp. 765-773, 2009.

[8] M. Weller, J. Felsberg, C. Hartmann et al., "Molecular predictors of progression-free and overall survival in patients with newly diagnosed glioblastoma: a prospective translational study of the German Glioma Network," Journal of Clinical Oncology, vol. 27, no. 34, pp. 5743-5750, 2009.

[9] S. E. Combs, S. Rieken, W. Wick et al., "Prognostic significance of IDH-1 and MGMT in patients with glioblastoma: one step forward, and one step back?" Radiation Oncology, vol. 6, pp. 115-119, 2011.

[10] D. J. Brat, D. J. Brat, R. G. Verhaak et al., "Comprehensive, integrative genomic analysis of diffuse lower-grade gliomas," The New England Journal of Medicine, vol. 372, no. 26, pp. 2481-2498, 2015.

[11] A. Cohen, S. Holmen, and H. Colman, "IDH1 and IDH2 mutations in gliomas," Curr Neurol Neurosci Rep, vol. 13, no. 5, pp. 345-357, 2013.

[12] J. Zhang, H. Liu, H. Tong et al., "Clinical applications of contrast-enhanced perfusion MRI techniques in gliomas: recent advances and current challenges," Contrast Media \& Molecular Imaging, vol. 2017, Article ID 7064120, 2017.

[13] W. L. Tan, W. Y. Huang, B. Yin, J. Xiong, J. S. Wu, and D. Y. Geng, "Can diffusion tensor imaging noninvasively detect IDH1 gene mutations in astrogliomas? A retrospective study of 112 cases," American Journal of Neuroradiology, vol. 35, no. 5, pp. 920-927, 2014.

[14] J. Xiong, W.-L. Tan, J.-W. Pan et al., "Detecting isocitrate dehydrogenase gene mutations in oligodendroglial tumors using diffusion tensor imaging metrics and their correlations with proliferation and microvascular density," Journal of Magnetic Resonance Imaging, vol. 43, no. 1, pp. 45-54, 2016.

[15] S. J. Price, K. Allinson, H. Liu et al., "Less invasive phenotype found in isocitrate dehydrogenase-mutated glioblastomas than in isocitrate dehydrogenase wild-type glioblastomas: a diffusion-tensor imaging study," Radiology, vol. 283, no. 1, pp. 215-221, 2017.

[16] J.-M. Hempel, S. Bisdas, J. Schittenhelm et al., "In vivo molecular profiling of human glioma using diffusion kurtosis imaging," Journal of Neuro-Oncology, vol. 131, no. 1, pp. 93-101, 2017.

[17] T. Liu, G. Cheng, X. Kang et al., "Noninvasively evaluating the grading and IDH1 mutation status of diffuse gliomas by threedimensional pseudo-continuous arterial spin labeling and diffusion-weighted imaging," Neuroradiology, vol. 60, no. 7, pp. 693-702, 2018.

[18] D. Roettger, J. L. Yuan, L. Mancini et al., "The role of diffusion tensor imaging for non-invasive IDH phenotyping in gliomas," Journal of Clinical Oncology, vol. 36, no. 15_suppl, 2018.
[19] Y. Tan, H. Zhang, X. Wang et al., "Comparing the value of DKI and DTI in detecting isocitrate dehydrogenase genotype of astrocytomas," Clinical Radiology, vol. 74, no. 4, pp. 314-320, 2019.

[20] W. Tan, J. Xiong, W. Huang, J. Wu, S. Zhan, and D. Geng, "Noninvasively detectingIsocitrate dehydrogenase 1gene status in astrocytoma by dynamic susceptibility contrast MRI," Journal of Magnetic Resonance Imaging, vol. 45, no. 2, pp. 492-499, 2017.

[21] K. Leu, G. A. Ott, A. Lai et al., "Perfusion and diffusion MRI signatures in histologic and genetic subtypes of WHO grade II-III diffuse gliomas," Journal of Neuro-Oncology, vol. 134, no. 1, pp. 177-188, 2017.

[22] S. Lee, S. H. Choi, I. Ryoo et al., "Evaluation of the microenvironmental heterogeneity in high-grade gliomas with IDH1/2 gene mutation using histogram analysis of diffusionweighted imaging and dynamic-susceptibility contrast perfusion imaging," Journal of Neuro-Oncology, vol. 121, no. 1, pp. 141-150, 2015.

[23] P. Kickingereder, F. Sahm, A. Radbruch et al., "IDH mutation status is associated with a distinct hypoxia/angiogenesis transcriptome signature which is non-invasively predictable with rCBV imaging in human glioma," Scientific Reports, vol. 5, 2015.

[24] A. Ulyte, V. K. Katsaros, E. Liouta et al., "Prognostic value of preoperative dynamic contrast-enhanced MRI perfusion parameters for high-grade glioma patients," Neuroradiology, vol. 58, no. 12, pp. 1197-1208, 2016.

[25] N. Anzalone, A. Castellano, M. Cadioli et al., "Brain gliomas: multicenter standardized assessment of dynamic contrastenhanced and dynamic susceptibility contrast MR images," Radiology, vol. 287, no. 3, pp. 933-943, 2018.

[26] P. S. Tofts, "Modeling tracer kinetics in dynamic Gd-DTPA MR imaging," Journal of Magnetic Resonance Imaging, vol. 7, no. 1, pp. 91-101, 1997.

[27] P. S. Tofts, G. Brix, D. L. Buckley et al., "Estimating kinetic parameters from dynamic contrast-enhanced t1-weighted MRI of a diffusable tracer: standardized quantities and symbols," Journal of Magnetic Resonance Imaging, vol. 10, no. 3, pp. 223-232, 1999.

[28] G. Brix, W. Semmler, R. Port, L. R. Schad, G. Layer, and W. J. Lorenz, "Pharmacokinetic parameters in CNS GdDTPA enhanced MR imaging," Journal of Computer Assisted Tomography, vol. 15, no. 4, pp. 621-628, 1991.

[29] K. S. S. Lawrence and T.-Y. Lee, "An adiabatic approximation to the tissue homogeneity model for water exchange in the brain: I. Theoretical derivation," Journal of Cerebral Blood Flow \& Metabolism, vol. 18, no. 12, pp. 1365-1377, 1998.

[30] T. S. Koh, L. H. Cheong, Z. Hou, and Y. C. Soh, "A physiologic model of capillary-tissue exchange for dynamic contrastenhanced imaging of tumor microcirculation," IEEE Transactions on Biomedical Engineering, vol. 50, no. 2, pp. 159-167, 2003.

[31] T. S. Koh, S. Bisdas, D. M. Koh, and C. H. Thng, "Fundamentals of tracer kinetics for dynamic contrast-enhanced MRI," Journal of Magnetic Resonance Imaging, vol. 34, no. 6, pp. 1262-1276, 2011.

[32] M. Inglese, K. L. Ordidge, L. Honeyfield et al., "Reliability of dynamic contrast-enhanced magnetic resonance imaging data in primary brain tumours: a comparison of Tofts and shutter speed models," Neuroradiology, vol. 61, no. 12, pp. 1375-1386, 2019.

[33] T. S. Koh, X. Y. Wu, L. H. Cheong, and C. C. T. Lim, “Assessment of perfusion by dynamic contrast-enhanced imaging 
using a deconvolution approach based on regression and singular value decomposition," IEEE Transactions on Medical Imaging, vol. 23, no. 12, pp. 1532-1542, 2004.

[34] T. S. Koh, L. H. D. Cheong, C. K. M. Tan, and C. C. T. Lim, “A distributed parameter model of cerebral blood-tissue exchange with account of capillary transit time distribution," Neurolmage, vol. 30, no. 2, pp. 426-435, 2006.

[35] M. D. Ruopp, N. J. Perkins, B. W. Whitcomb, and E. F. Schisterman, "Youden Index and optimal cut-point estimated from observations affected by a lower limit of detection," Biometrical Journal, vol. 50, no. 3, pp. 419-430, 2008.

[36] M. Law, S. Yang, J. S. Babb et al., "Comparison of cerebral blood volume and vascular permeability from dynamic susceptibility contrast-enhanced perfusion MR imaging with glioma grade," AJNR. American Journal of Neuroradiology, vol. 25, no. 5, pp. 746-755, 2004.

[37] Z. M. Bhujwalla, D. Artemov, K. Natarajan, M. Solaiyappan, P. Kollars, and P. E. G. Kristjansen, "Reduction of vascular and permeable regions in solid tumors detected by macromolecular contrast magnetic resonance imaging after Treatment with antiangiogenic agent TNP470," Clinical Cancer Research, vol. 9, no. 1, pp. 355-362, 2003.

[38] H. Degani, M. C. Dadiani, L. Bogin, and E. F. Haran, "Magnetic resonance imaging of tumor vasculature," Thrombosis and Haemostasis, vol. 89, no. 1, pp. 23-33, 2003.

[39] P. Vajkoczy and M. D. Menger, "Vascular microenvironment in gliomas," Journal of Neuro-Oncology, vol. 50, no. 1/2, pp. 99-108, 2000.

[40] J.-M. Hempel, J. Schittenhelm, U. Klose et al., "In vivo molecular profiling of human glioma," Clinical Neuroradiology, vol. 29, no. 3, pp. 479-491, 2019.

[41] D. M. McDonald and P. L. Choyke, "Imaging of angiogenesis: from microscope to clinic," Nature Medicine, vol. 9, no. 6, pp. 713-725, 2003.

[42] D. M. McDonald and P. Baluk, "Significance of blood vessel leakiness in cancer," Cancer Research, vol. 62, no. 18, pp. 5381-5385, 2002.

[43] K. L. Li, X. P. Zhu, D. R. Checkley et al., "Simultaneous mapping of blood volume and endothelial permeability surface area product in gliomas using iterative analysis of firstpass dynamic contrast enhanced MRI data," The British Journal of Radiology, vol. 76, no. 901, pp. 39-50, 2003.

[44] Y. Lu, W. Peng, J. Song et al., "On the potential use of dynamic contrast-enhanced (DCE) MRI parameters as radiomic features of cervical cancer," Medical Physics, vol. 46, no. 11, pp. 5098-5109, 2019.

[45] X. Wang, W. X. Lin, Y. T. Mao et al., "A comparative study of two-compartment exchange models for dynamic contrastenhanced mri in characterizing uterine cervical carcinoma," Contrast Media \& Molecular Imaging, vol. 2019, Article ID 3168416, 13 pages, 2019.

[46] H. J. van de Haar, S. Burgmans, J. F. A. Jansen et al., "Bloodbrain barrier leakage in patients with early Alzheimer disease," Radiology, vol. 281, no. 2, pp. 527-535, 2016.

[47] S. J. Mills, D. du Plessis, P. Pal et al., "Mitotic activity in glioblastoma correlates with estimated extravascular extracellular space derived from dynamic contrast-enhanced MR imaging," American Journal of Neuroradiology, vol. 37, no. 5, pp. 811-817, 2016.

[48] S. P. Sourbron and D. L. Buckley, "On the scope and interpretation of the Tofts models for DCE-MRI," Magnetic Resonance in Medicine, vol. 66, no. 3, pp. 735-745, 2011.
[49] R. A. Cairns and T. W. Mak, "Oncogenic isocitrate dehydrogenase mutations: mechanisms, models, and clinical opportunities," Cancer Discovery, vol. 3, no. 7, pp. 730-741, 2013.

[50] D. Russel and L. Rubinstein, Pathology of Tumors of the Nervous System, Williams \& Wilkins, Baltimore, Maryland, 1989.

[51] C. Hartmann, J. Meyer, J. Balss et al., "Type and frequency of IDH1 and IDH2 mutations are related to astrocytic and oligodendroglial differentiation and age: a study of 1,010 diffuse gliomas," Acta Neuropathologica, vol. 118, no. 4, pp. 469-474, 2009.

[52] N. A. Obuchowski, "Sample size tables for receiver operating characteristic studies," American Journal of Roentgenology, vol. 175 , no. 3, pp. 603-608, 2000.

[53] J. Carpenter and J. Bithell, "Bootstrap confidence intervals: when, which, what? A practical guide for medical statisticians," Statistics in Medicine, vol. 19, no. 9, pp. 1141-1164, 2000. 